\title{
Мезопористый кремнезем МСМ-48, особенности его синтеза и перспективы применения
}

\author{
® 2019 Нефедова Т.Н. ${ }^{1,2}$, Ресснер Ф. ${ }^{1}$, Селеменев В.Ф. ${ }^{3}$ \\ ${ }^{1}$ Ольденбургский университет имени Карла фон Осеикого, Ольденбург, Германия \\ ${ }^{2}$ Воронежский государственный технический университет, Воронеж, Россия \\ ${ }^{3}$ Воронежский государственный университет, Воронеж, Россия
}

Поступила в редакцию 27.11.2019 г.

DOI: $10.17308 /$ sorpchrom.2020.20/2377

Аморфный кремнезем - синтетический материал, обладающий уникальными свойствами, незаменимый во многих направлениях современной мировой индустрии, в медицине и косметологии. Аморфный диоксид кремния представляет собой систему пор разного размера: макро- (d>50 нм), ме3о- $(2<\mathrm{d}<50$ нм) и микропоры $(\mathrm{d}<2$ нм $)$.

Успешно синтезированная в 1992 году компанией Mobil группа мезопористых кремнеземов с узким распределением пор по размеру и развитой площадью поверхности (>1000 м²/г), имеющих общее название M 41S позволила решить проблему неоднородности пор аморфного диоксида кремния. Важными представителями группы являются МСМ-41 с гексагональной двухмерной структурой пор, МСМ-48 с трехмерной кубической и МСМ-50 со слоистой. Кубическая структура МСМ-48 организована таким образом, что создает условия для удобного массопереноса молекул и сокращает диффузионные ограничения, делая этот материал устойчивым к закупорке пор.

В настоящей статье рассмотрены особенности синтеза МСМ-48 и перспективы его применения. Синтез мезопористого МСМ-48 зависит от многих факторов - температуры, времени реакции, скорости перемешивания, $\mathrm{pH}$ реакционной среды, соотношения исходных компонентов, природы растворителя, природы прекурсора $\mathrm{Si}$, длины цепочки КПАВ (катионные поверхностно-активные вещества) - и может осуществляется двумя путями: гидротермально- или при комнатной температуре.

Особенности структуры мезопористого кремнезема МСM-48 делает этот материал привлекательным для применения в процессах катализа, как в качестве катализатора (Si-MCM-48), так и основы для катализатора (Al-MCM-48, Pt/MCM-48, ZnO-CuO/MCM-48), в процессах сорбции и разделения (МСM-48 модифицированный 3-меркаптопропилтриметоксисиланом, Al-MCM-48 с бимодальной системой пор) и в микрофлюидике в роли наностуктурированной ион-секлективной мембраны (слои МСМ-48, нанесеные на макропористые подложки из $\alpha$-оксида алюминия и микросит из нитрида кремния).

Ключевые слова: мезопористый кремнезем, МСМ-48, синтез, перспективы применения.

Аморфный кремнезем - синтетический материал, обладающий уникальными свойствами, незаменимый во многих направлениях современной мировой индустрии. Безвредность аморфного диоксида кремния для живых организмов создает возможность для применения этого материала в медицине, косметологии и пищевой промышленности, а высокая удельная поверхность, пористость и нейтральность позволяют ему изменять свойства других веществ - текучесть и сыпучесть материалов, термодинамические, кинетические и трибологические свойства.

Аморфный диоксид кремния представляет собой систему пор разного размера: макро- $(\mathrm{d}>50$ нм $)$, мезо- $(2<\mathrm{d}<50$ нм) и микропоры $(\mathrm{d}<2$ нм $)[1,2]$. По мнению авторов [3], аморфность стенок пор может ограничивать применимость этого материала, например, в катализе, поэтому предпринимались попытки по укреплению тонких 
стенок мезопор пористого $\mathrm{SiO}_{2}$ за счет кристаллизации, процесс оказался трудоемким для исполнения.

В 1992 году компанией Mobil была успешно синтезирована группа мезопористых кремнеземов с узким распределением пор по размеру и развитой площадью поверхности (>1000 м²/г), имеющих общее название M 41S, что позволило решить проблему неоднородности пор аморфного диоксида кремния [4-6]. Важными представителями группы являются МСM-41 с гексагональной двухмерной структурой пор, МСМ-48 с трехмерной кубической и МСМ-50 со слоистой. Структурные свойства этих мезопористых материалов определяют их применение, так, например, они могут быть использованы в качестве подложки (носителя) для катализатора $[7,8]$, в процессах разделения $[9,10]$ или же в качестве каркаса для синтеза наноуглерода [11].

Синтез материалов осуществлялся темплатным методом. В качестве темплат использовались катионные поверхностно-активные вещества (КПАВ) содержащие длинные алкильные цепи (10-20 атомов углерода), а именно соединения четвертичного аммония - цетилтриметиламмония бромид (СТАВ) или цетилтриметиламмония хлорид $(\mathrm{CTACl})$ и т.п. Исследователями Mobil group было предложено два механизма синтеза (рис.1). Согласно первому механизму КПАВ образуют лиотропную жидкокристаллическую фазу, которая служит в качестве каркаса для будущей структуры. Далее в реакционную смесь вводят кремнийсодержащий компонент. Происходит протекание «золь-гель» процесса: часть анионов прекурсора $\mathrm{Si}$, образующиеся в системе на этапе реакции гидролиза взаимодействуют с катионами полярной части КПАВ, остальная часть взаимодействует с катионами водорода, образуя гидроксосоединения, далее эти гидроксосоединения конденсируются между собой, формируя неорганический полимерный слой вокруг сурфактанта (ПАВ). В соответствии со вторым механизмом частицы КПАВ инициируются к образованию структурного каркаса присутствующими в реакционной смеси частицами кремнийсодержащего вещества. Авторы [12] считают, что основной действующей силой в этом случае выступают кулоновские взаимодействия, в результате чего беспорядочно расположенные мицеллы КПАВ окружаются двойным или тройным монослоем кремнезема. Далее мицеллы, окруженные слоями кремнезема, стараются занять наиболее энергетически выгодное положение и упаковываются в упорядоченный мезопористый агрегат - происходит конденсация олигомеров кремнезема. Последующая конденсация между агрегатами протекает под действием температуры с формированием финальной структуры.

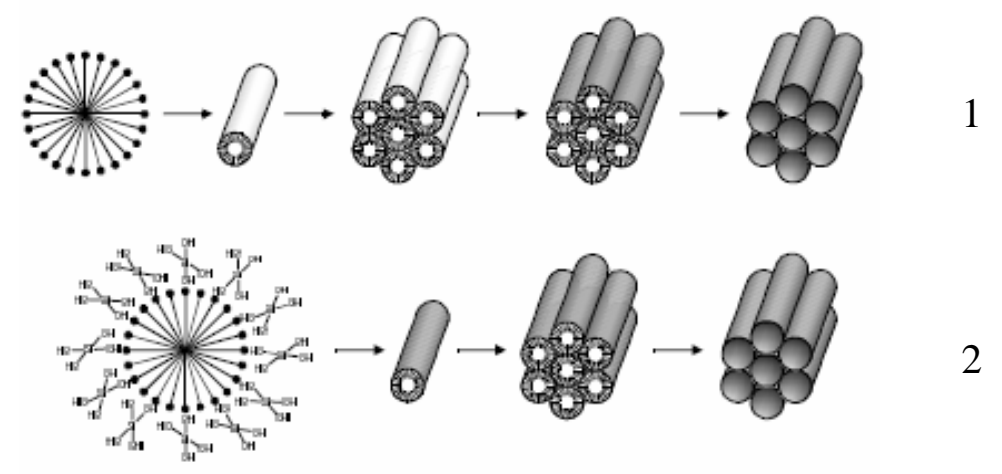

Рис. 1. Механизм образования мезопористых материалов [4-6].

В настоящее время существует много работ, предлагающих другие варианты механизмов образования мезопористых кремнеземов, и посвященных попыткам объ- 
яснить благодаря какому из видов взаимодействий частицы КПАВ вступают в реакцию с частицами кремнийсодержащего компонента [13-17]

Условия получения для каждого из материалов группы M 41S различны и оказывают значительное влияние на формирование типа структуры [18-23]. До определенного момента интерес в основном был сфокусирован на МСМ-41 [24-26], вероятнее всего, из-за сложности проведения синтеза мезопористых материалов с другим типом структуры, например, МСМ-48.

Сильно разветвленная и переплетенная кубическая структура МCM-48 с $l a \overline{3} d$ симметрией (рис.2) организована таким образом, что позволяет избежать закупорки пор молекулами реагента, тем самым расширяя область его применения относительно двумерных мезопористых аналогов [27].

Синтез мезопористого МСМ-48 зависит от многих факторов - температуры, времени реакции, скорости перемешивания, $\mathrm{pH}$ реакционной среды, соотношения исходных компонентов, природы растворителя, природы прекурсора $\mathrm{Si}$, длины цепочки КПАВ - и может осуществляется двумя путями: гидротермально- или при комнатной температуре [5, 13, 28-37].

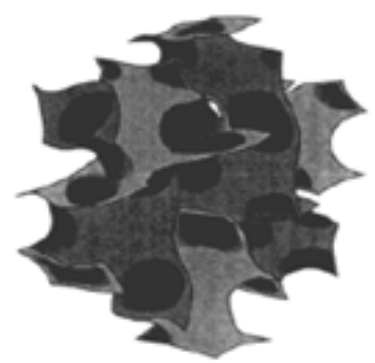

a)

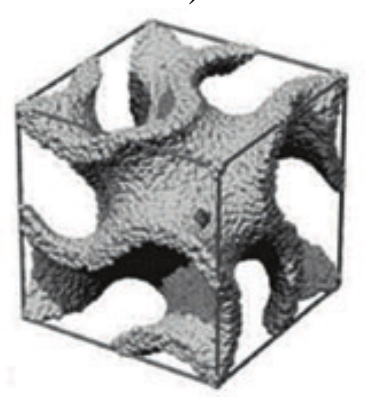

б)

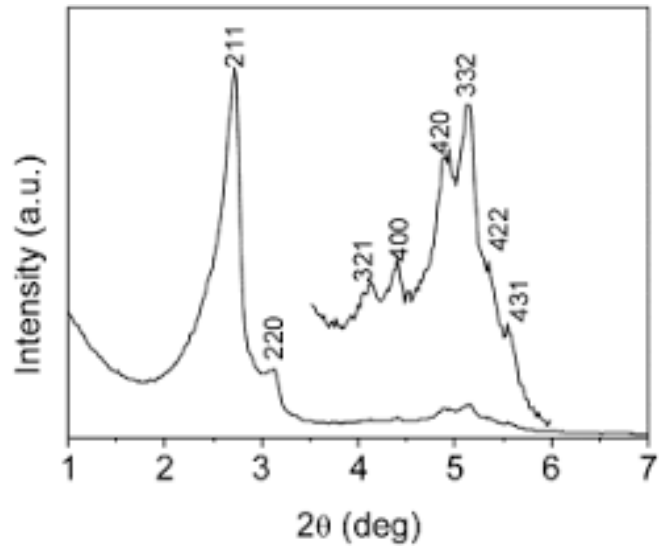

в)

Рис. 2. Фрагмент структуры (а), элементарная ячейка (б) и рентген-дифрактограмма МСМ-48 (в) [27].

Первые независимые и согласующиеся между собой сообщения о получении $l a \overline{3} d$ структуры относятся к промежутку времени между 1989-1992 [5, 28]. Далее авторами [13] было объяснено образование 3D структуры и установлено, что эта возможность создается при длине углеродной цепи КПАВ от 8 до 20 атомов С, высоких значениях $\mathrm{pH}$ и соотношении компонентов $\mathrm{CTA} / \mathrm{SiO}_{2}$. Синтез осуществлялся из реакционной смеси составом 1 M TEOS $\left.\left(\mathrm{C}_{2} \mathrm{H}_{5} \mathrm{O}\right)_{4} \mathrm{Si}\right): 0.25 \quad \mathrm{M} \quad \mathrm{Na}_{2} \mathrm{O}: 0.65 \quad \mathrm{M}$ $\mathrm{CTACl} / \mathrm{CTAB}\left(\mathrm{C}_{16} \mathrm{H}_{33}\left(\mathrm{CH}_{3}\right)_{3} \mathrm{NCl} / \mathrm{C}_{16} \mathrm{H}_{33}\left(\mathrm{CH}_{3}\right)_{3} \mathrm{NBr}\right)$ : 0.62M H $\mathrm{H}_{2} \mathrm{O}$ при температуре 383 К гидротермальным методом в тефлоновом автоклаве покрытым ПТФЭ (реактор периодического действия) в течение 3 дней. 
Позже было выявлено влияние времени протекания реакции [29]. Найдено, что при вышеописанных условиях формирование правильной кубической структуры возможно только по истечении десяти дней.

Положительные результаты отмечены и в синтезе МСМ-48 при комнатной температуре (1 M TEOS: $12.5 \mathrm{M} \mathrm{NH}_{3} \cdot \mathrm{H}_{2} \mathrm{O}: 54 \mathrm{M} \mathrm{EtOH}\left(\mathrm{C}_{2} \mathrm{H}_{5} \mathrm{OH}\right): 0.4 \mathrm{M}$ CTAB: $417 \mathrm{M}$ $\mathrm{H}_{2} \mathrm{O}$; 0.41M CTAB: $11 \mathrm{M} \mathrm{NH} \mathrm{H}_{3} \cdot \mathrm{H}_{2} \mathrm{O}$ : 1.0M TEOS: 53M EtOH: $344 \mathrm{M} \mathrm{H} \mathrm{H}_{2} \mathrm{O}$ ) [29-32]. Ocновными факторами влияющими на формирование кубической структуры в этом случае являются время реакции и скорость перемешивания [29, 31, 32]. В качестве наиболее оптимальных параметров приведены следующие: время реакции 20 минут, скорость перемешивания 300 об/мин или без перемешивания после добавления прекурсора Si [32].

Использование различных источников $\mathrm{SiO}_{2}$ в качестве прекурсора $\mathrm{Si}$ - коллоидный $\mathrm{SiO}_{2}\left(99 \% \mathrm{SiO}_{2}\right)$, этилсиликат $\left(40 \% \mathrm{SiO}_{2}\right)$ и золь кремнезема $\left(40 \% \mathrm{SiO}_{2}\right)$ - показало, что тип источника диоксида кремния контролирует толщину стенок пор и, следовательно, обуславливает стабильность структуры МСМ-48 [31, 33].

Рядом авторов указывалось на возможность процессов трансформации структуры МСМ-41 в МСМ-48 при изменении количества этанола в реакционной смеси. Трансформация наблюдается как для гидротермального метода получения (8.4 M TEOS: 1M CTAB: 2.1M Na $2 \mathrm{O}: 1108.9 \mathrm{M} \mathrm{H}_{2} \mathrm{O}: \mathrm{xM}$ EtOH:) [34, 35], так и при комнатной температуре (1M TEOS: 0.3M CTAB: $11 \mathrm{M} \mathrm{NH}_{3} \cdot \mathrm{H}_{2} \mathrm{O}: \mathrm{xM}$ EtOH: yM H2O) [36]. Некоторые авторы сообщают [21], что возрастание количества алкоголя в системе может приводить к преобразованию типа мезоструктуры в ряду $\mathrm{MCM}-41 \rightarrow \mathrm{MCM}-$ $48 \rightarrow$ слоистая $\rightarrow$ радиально гексагональная.

В большинстве работ, описанных выше, для синтеза МСМ-48 в качестве исходных компонентов используются такие вещества, как CTAB (CTACl) в роли KПAB, TEOS как прекурсор Si и этанол - направляющий структуру агент. Однако, группой авторов достигнут достаточно хороший результат с применением блок сополимера Р123 (КПАВ), силиката натрия (источник кремния) и n-бутанола (структуронаправляющее вещество) [37]. Полученный при таких условиях МСМ-48 обладал теми же структурными характеристиками и свойствами, что и синтезированный из другого состава.

Ранее в статье упоминалось, что кубическая структура МСМ-48 организована таким образом, что создает условия для удобного массопереноса молекул и сокращает диффузионные ограничения, делая этот материал устойчивым к закупорке пор и привлекательным для каталитического и сорбционного применения.

Все более востребованными в настоящее время становятся упорядоченные мезопористые материалы неорганической природы, способные сохранять свои свойства при работе в условиях повышенных температур и в органических растворителях $[38,39]$. Например, дальнейшая пост-синтетическая обработка кальцинированного MCM-48 сверхкритическим $\mathrm{CO}_{2}\left(\mathrm{sc}-\mathrm{CO}_{2}\right)$ и небольшим количеством втор-бутоксида $\mathrm{Al}$ (Al sec-butoxide) значительно улучшает гидротермальную стабильность полученного материала, что создает предпосылки для его использования в катализе [40].

$\mathrm{ZnO}-\mathrm{CuO} / \mathrm{MCM}-48$ катализаторы проявляют фотокаталитическую активность в реакциях переработки салициловой кислоты и метиленового синего, содержащиеся в органических отходах производства [41].

Была осуществлена попытка применения МСМ-48, импрегнированного $\mathrm{Pt}$ (Pt/MCM-48), в процессе селективного каталитического восстановления $\mathrm{NO}_{\mathrm{X}}$, и проведено сравнение каталитической активности с Pt/MCM-41 (катализаторы получены одним методом). Исследования показали, большую реакционную способность $\mathrm{Pt} / \mathrm{MCM}-48$ по причине импрегнирования на его поверхности сразу двух видов окси-

Нефедова и др. / Сорбционные и хроматографические процессы. 2020. Т. 20. № 1. С. 31-39 
дов платины $\mathrm{Pt}^{2+}(\mathrm{PtO})$ and the $\mathrm{Pt}^{4+}\left(\mathrm{PtO}_{2}\right)$ и наиболее лучшего их распределения в 3D структуре кремнезема [42].

Химическая модификация МСМ-48 прививкой 3-меркаптопропилтриметоксисилана сообщает высокую сорбционную способность материалу в отношении ионов $\mathrm{Pb}^{2+}$, что позволяет его использовать для очистки сточных вод гальванического производства от тяжелых металлов [43].

Материалы с бимодальной и мультимодальной системой пор на основе упорядоченных мезопористых кремнеземов могут повысить эффективность процессов разделения веществ [44-47]. Так авторами [48] пост-синтетической модификацией был получен бимодальный мезопористый Al-MCM-48 содержащий упорядоченную непрерывную $l a \overline{3} d$ кубическую систему пор МСМ-48 и нерегулярную систему пор с преобладающими размерами 26 и $38 \AA$.

Al-MCM-48 обладает не только сорбционными свойствами, но и проявляет каталитическую активность при крекинге продуктов пиролиза отходов пластмасс, например, линейного полиэтилена низкой плотности (LLDPE) [49].

Применение МСM-48 в качестве наностуктурированной ион-селективной мембраны (слои МСМ-48, нанесенные на макропористые подложки из $\alpha$-оксида алюминия и микросит из нитрида кремния) в микрофлюидике также дает хорошие результаты [50].

Выше представленная информация позволяет сделать заключение о том, что мезопористый кремнезем МСМ-48 является перспективным и востребованным материалом для промышленности, экологии, медицины и биотехнологии, способным увеличить эффективность и экологичность таких процессов, как катализ, сорбция и разделение, микрофлюидика, переработка и очистка отходов.

Проект выполнен при финансовой поддержке Германской службы академических обменов (DAAD) в рамках программы «Михаил Ломоносов» и по проекту Министерства науки и высшего образования РФ государственного заказа No. 4.13418.2019/13.2 (730000Ф.99.1.БB10AA00006).

\section{Список литературы}

1. Кузнецова Т. Ф., Ратько А. И., Еременко С. И. // Журнал физической химии. 2012. Т. 86. № 10. C. 1730-1734.

2. IUPAC Recommendations // Pure Appl. Chem. 1985. Vol. 57. No 4. pp. 603-619.

3. Yang P., Zhao D., Margolese D.I., Chmelka B.F. et al. // Chem. Mater. 1999. Vol. 11. pp. 2813-2826.

4. Kresge C.T., Leonowicz M.E., Roth W.J., Vartuli J.C. et al. // Nature. 1992. Vol. 359. pp. 710-712.

5. Beck J.S., Vartuli J.C., Roth W.J., Leonowicz M.E. et al. // J. Am. Chem. Soc. 1992. Vol. 114. pp. 10834-10843.

6. Huo Q., Margolese D. I., Ciesla U., Feng P. et al. // Nature. 1994. Vol. 368. pp. 317-321.

7. Cavalleri M., Hermann K., Knop-Gericke A., Havecker M. et al. // J. Catal. 2009. Vol. 262. Iss. 2. pp. 215-223.
8. Ghita D., Rosca P., Stanica Ezeanu D. // Revista de Chimie. 2012. Vol. 63. Iss. 10. pp. 1056-1061.

9. Hoang V.T., Huang Q., Kaliaguine S. // Langmuir. 2005. Vol. 21. Iss. 5, pp. 2051-2057.

10.Han Y. J., Stuck G. D., Butler A. // J. Am. Chem. Soc. 1999. Vol. 121. pp. 9897-9898.

11.Ryoo R., Ko C.H., Kruk M., Antochshuk V. et al. // Journal Phys. Chem B. 2000. Vol. 104. Iss. 48. pp. 11465-11471.

12. Chen C.Y., Burkett S.L., Li H.X., Davis M.E. // Microporous Mater. Vol. 2. Iss. 1. pp. 27-34.

13.Monnier A., Schüth F., Huo Q., Kumar D. et al. // Science. 1993. Vol. 261. Iss. 5126. pp. 1299-1303.

14.Firouzi A., Atef F., Oertli A.G., Stucky G.D. et al. // J. Am. Chem. Soc. 1997. Vol. 119. Iss. 15. pp. 3596-3610. 
15.Inagaki S., Guan S., Fukushima Y., Ohsuma T. et al. // J. Am. Chem. Soc. 1999. Vol. 121. Iss. 41. pp. 9611-9614.

16.Guan S., Inagaki S., Ohsuma T., Terasaki O. // J. Am. Chem. Soc. 2000. Vol. 122. Iss. 23. pp. 5660-5661.

17.Tanev P.T., Pinnavaia T.J. // Science. 1995. Vol. 267. Iss. 519. pp. 865-867.

18.Hoa M.L.K., Lu M., Zhang Y. // Adv. Colloid Interf. Sci. 2006. Vol. 121. Iss. 1-3. pp. 923.

19.Liang Y., Erichsen E.S., Hanzlik M., Anwander R. // Chem. Mater. 2008. Vol. 20. Iss. 4. pp. 1451-1458.

20.Xia Y., Mokaya R., Titman J.J. // J. Phys. Chem. B. 2004. Vol. 108. Iss. 31. pp. 1136111367.

21.Liu S., Cool P., Collart O., Van Der Voort P. et al. // J. Phys. Chem. B. 2003. Vol. 107. Iss. 38. pp. 10405-10411.

22. Araki S., Doi H., Sano Y., Tanaka S. et al. // J. Colloid Interf. Sci. 2009. Vol. 339. Iss. 2. pp. 382-389.

23.Fu X.-C., Chen X., Wang J., Liu J.-H. et al. // Electrochim. Acta. 2010. Vol. 56. Iss.1. pp. 102-107.

24.Huo Q., Margolese D.I., Stucky G.D. // Chem. Mater. 1996. Vol. 8. Iss.5. pp. 11471160.

25.Ciesla U., Grün M., Isajeva T., Kurganov A.A. et al. // In Access in Nanoporous Materials, Pinnavaia T.J., Thorpe M.F. Eds. Plenum Press. New York. 1995. p 231.

26.Grün M., Unger K.K., Matsumoto A., Tsutsumi K. // In Charaterization of Porous Solids IV, Eds. The Royal Society of Chemistry: Cambridge. 1997. p 81.

27.Gallis K. W., Landry C.C. // Chem. Mater. 1997. Vol. 9. Iss. 10. pp. 2035-2038.

28. Auvray X., Petipas C., Anthore R., Rico I. et al. // J. Phys. Chem. 1989. Vol. 93. Iss. 21. pp. 7458-7464.

29.Schumacher K., Ravikovitch P.I., Du Chesne A., Neimark A.V. et al. // Langmuir. 2000. Vol. 16. Iss. 10. pp. 4648-4654.

30.Schumacher K., Grün M., Unger K.K. // Microporous Mesoporous Mater. 1999. Vol. 27. Iss. 2-3. pp. 201-206.

31. Matei D., Cursaru L., Mihai S. // Digest Journal of Nanomaterials and Biostructures. 2016. Vol. 11. No 1. pp. 271-276.

32.Boote B., Subramanian H., Ranjit K.T. // Chemical communications. 2007. No.43. pp. 4543-4545.
33.Gaydhankar T.R., Taralkar U.S., Jha R.K., Joshi P.N. et al. // Catalysis Communication. 2005. Vol. 6. Iss. 5. pp. 361-366.

34.Gallis K.W., Landry C.C. // Chemistry of Materials. 1997. Vol. 9. Iss. 10. pp. 2035-2038.

35.Xia Y., Mokaya R., Titman J.J. // J. Phys. Chem. B. 2004. Vol. 108. Iss. 31. pp. 1136111367.

36.Qin L., Zhao H., Wang H., Liu S. // $A d$ vanced Materials Research. 2011. Vol. 306307. pp. 1420-1424.

37.Kim T.-W., Kleitz F., Paul B., Ryoo R. // J. Am. Chem. Soc. 2005. Vol. 127. Iss. 20. pp. 7601-7610.

38.Gavalas G.R., Megiris C.E., Nam S.W. // Chem. Eng. Sci. 1989. Vol. 44. Iss. 9. pp. 18291835.

39.Tsapatsis M., Kim S., Nam S.W., Gavalas G. // Ind. Eng. Chem. Res. 1991. Vol. 30. Iss. 9. pp. 2152-2159.

40.Wang K., Lin Y., Morrisa M.A., Holmes J.D. // J. Mater. Chem. 2006. Vol. 16. Iss. 41. pp. 4051-4057.

41.Duan Y., Shen Y. // Water Science \& Technology. 2017. Vol. 76. Iss. 1. pp. 172-181.

42.Kim D.-J., Cho J.-K., Jang J.-H., Lee S.-C. et al. // Applied Catalysis A: General. 2005. Vol. 286. Iss. 1. pp. 36-43.

43.Taba P., Mustafa R.D.P., Ramang L.M., Kasim A.H. // Journal of Physics: Conf. Series. 2018. Vol. 979. Conference 1. ID. 012058.

44. Yuan Z.Y., Wang J.Z., Zhang Z.L., Chen T.H., Li H.X. // Microporous Mesoporous Mater. 2001. Vol. 43. Iss. 2. pp. 227-236.

45.Sun J.H., Shan Z., Maschmeyer T., Coppens M.O. // Langmuir. 2003. Vol. 19. Iss. 20. pp. 8395-8402.

46. Grimes B.A., Lüdtke S., Unger K.K., Liapis A.I. // Journal of Chromatography A. 2002.

Vol. 979. Iss. 1-2. pp. 447-466.

47.Liapis A.I., Meyers J.J., Crosser O.K. // Journal of Chromatography A. 1999. Vol. 865. Iss. 1-2. pp. 13-25.

48.Guan L.C., Nur H., Endud S. // Journal of Physical Science. 2006. Vol. 17. Iss. 1. pp. 6575.

49.Jeon J.-K., Park H. J., Yim J.-H., Kim J. M. et al. // Solid State Phenomena. 2007. Vol. 124126. pp. 1757-1760.

50.Schmuhl R., Chowdhury S.R., ten Elshof J.E., van den Berg A. et al. // Journal of Sol-Gel Science and Technology. 2004. Vol. 31. Iss. 1-3. pp. 249-252. 


\title{
Mesoporous silica MCM-48: synthesis and application prospects
}

\author{
Nefedova Tatiana N. ${ }^{1,2}$, Roessner Frank ${ }^{1}$, Selemenev Vladimir F. ${ }^{3}$ \\ ${ }^{1}$ Carl von Ossietzky University, Oldenburg, Germany \\ ${ }^{2}$ Voronezh State Technical University, Voronezh, Russia \\ ${ }^{3}$ Voronezh State University, Voronezh, Russia
}

\begin{abstract}
Amorphous silica is an inorganic material with unique properties. It has become a key material in various areas of global industry, medicine, and cosmetology. Amorphous silicon dioxide consists of an arrangement of pores of various size: macro- $(\mathrm{d}>50 \mathrm{~nm})$, meso- $(2<\mathrm{d}<50 \mathrm{~nm})$, and micropores $(\mathrm{d}<2 \mathrm{~nm})$.

In 1992, Mobil Oil Corporation developed a group of mesoporous materials named M 41S with a sharp pore distribution on the surface and a large pore volume $\left(>1000 \mathrm{~m}^{2} / \mathrm{g}\right)$ thus addressing the issue of different-size pores of amorphous silicon dioxide. The key materials in this group are MCM-41 with a hexagonal two-dimensional pore system, MCM-48 with a cubic pore system, and MCM-50 with a layered pore system. The cubic pore structure of MCM- 48 creates the conditions for convenient mass transfer of molecules which reduces diffusion restrictions and makes this material resistant to clogging of pores.

The paper focuses on the specific features of MCM- 48 synthesis, as well as its application prospects. The synthesis of the mesoporous silica MCM-48 can be carried out in two modes: hydrothermal synthesis and room-temperature synthesis. The process itself is determined by a number of factors, including the reaction temperature, the reaction time, the stirring rate, $\mathrm{pH}$, the ratio of the initial components, the nature of the solvent, the nature of the Si precursor, and the length of the surfactant chain.

Due to its structure, the mesoporous silica MCM-48 has a wide range of applications: in catalysis processes both as a catalyst (Si-MCM-48) and as a catalyst base (Al-MCM-48, Pt/MCM-48, ZnO$\mathrm{CuO} / \mathrm{MCM}-48$ ); in sorption and separation processes (MCM-48 modified by 3mercaptopropyltrimethoxysilane, Al-MCM-48 with a bimodal pore system); in microfluidics as a nanostructured ion-selective membrane (MCM-48 layers deposited on macroporous $\alpha$-aluminium oxide substrate and silicon nitride microsieves).
\end{abstract}

Keywords: mesoporous silica, MCM-48, synthesis, application prospects.

\section{References}

1. Kuznetsova T.F., Ratko A.I., Eremenko S.I., Russian Journal of Physical Chemistry A, 2012, Vol. 86, No 10, pp. 1730-1734.

2. IUPAC Recommendations, Pure Appl. Chem., 1985, Vol. 57, No 4, pp. 603-619.

3. Yang P., Zhao D., Margolese D.I., Chmelka B.F. et al., Chem. Mater., 1999, Vol. 11, pp. 2813-2826.

4. Kresge C.T., Leonowicz M.E., Roth W.J., Vartuli J.C. et al., Nature, 1992, Vol. 359, pp. 710-712.

5. Beck J.S., Vartuli J.C., Roth W.J., Leonowicz M.E. et al., J. Am. Chem. Soc., 1992, Vol. 114, pp. 10834-10843.

6. Huo Q., Margolese D.I., Ciesla U., Feng P. et al., Nature, 1994, Vol. 368, pp. 317-321.

7. Cavalleri M., Hermann K., Knop-Gericke A., Havecker M. et al., J. Catal., 2009, Vol. 262, Iss. 2, pp. 215-223.

8. Ghita D., Rosca P., Stanica Ezeanu D., Revista de Chimie, 2012, Vol. 63, Iss. 10, pp. 1056-1061.
9. Hoang V.T., Huang Q., Kaliaguine S., Langmuir, 2005, Vol. 21, Iss. 5, pp. 2051-2057.

10.Han Y. J., Stuck G. D., Butler A., J. Am. Chem. Soc., 1999, Vol. 121, pp. 9897-9898.

11. Ryoo R., Ko C.H., Kruk M., Antochshuk V. et al., Journal Phys. Chem B., 2000, Vol. 104, Iss. 48, pp. 11465-11471.

12.Chen C.Y., Burkett S.L., Li H.X., Davis M.E., Microporous Mater., Vol. 2, Iss. 1, pp. 27-34.

13. Monnier A., Schüth F., Huo Q., Kumar D. et al., Science, 1993, Vol. 261, Iss. 5126, pp. 1299-1303.

14.Firouzi A., Atef F., Oertli A.G., Stucky G.D. et al., J. Am. Chem. Soc., 1997, Vol. 119, Iss. 15, pp. 3596-3610.

15.Inagaki S., Guan S., Fukushima Y., Ohsuma T. et al., J. Am. Chem. Soc., 1999, Vol. 121, Iss. 41, pp. 9611-9614.

16.Guan S., Inagaki S., Ohsuma T., Terasaki O., J. Am. Chem. Soc., 2000, Vol. 122, Iss. 23, pp. 5660-5661. 
17.Tanev P.T., Pinnavaia T.J., Science, 1995, Vol. 267, Iss. 519, pp. 865-867.

18.Hoa M.L.K., Lu M., Zhang Y., Adv. Colloid Interf. Sci., 2006, Vol. 121, Iss. 1-3, pp. 9-23.

19.Liang Y., Erichsen E.S., Hanzlik M., Anwander R., Chem. Mater., 2008, Vol. 20, Iss. 4, pp. 1451-1458.

20.Xia Y., Mokaya R., Titman J.J., J. Phys. Chem. B, 2004, Vol. 108, Iss. 31, pp. 1136111367.

21.Liu S., Cool P., Collart O., Van Der Voort P. et al., J. Phys. Chem. B, 2003, Vol. 107, Iss. 38, pp. 10405-10411.

22. Araki S., Doi H., Sano Y., Tanaka S. et al., J. Colloid Interf. Sci., 2009, Vol. 339, Iss. 2, pp. 382-389.

23.Fu X.-C., Chen X., Wang J., Liu J.-H. et al., Electrochim. Acta, 2010, Vol. 56, Iss. 1, pp. 102-107.

24.Huo Q., Margolese D.I., Stucky G.D., Chem. Mater., 1996, Vol. 8, Iss. 5, pp. 11471160.

25.Ciesla U., Grün M., Isajeva T., Kurganov A.A. et al., In Access in Nanoporous Materials, Pinnavaia T.J., Thorpe M.F. Eds. Plenum Press. New York, 1995, p 231.

26.Grün M., Unger K.K., Matsumoto A., Tsutsumi K., In Charaterization of Porous Solids IV, Eds. The Royal Society of Chemistry: Cambridge, 1997, p 81.

27.Gallis K.W., Landry C.C., Chem. Mater., 1997, Vol. 9, Iss. 10, pp. 2035-2038.

28. Auvray X., Petipas C., Anthore R., Rico I. et al., J. Phys. Chem., 1989, Vol. 93, Iss. 21, pp. 7458-7464.

29. Schumacher K., Ravikovitch P.I., Du Chesne A., Neimark A.V. et al., Langmuir, 2000, Vol. 16, Iss. 10, pp. 4648-4654.

30. Schumacher K., Grün M., Unger K.K., Microporous Mesoporous Mater., 1999, Vol. 27, Iss. 2-3, pp. 201-206.

31. Matei D., Cursaru L., Mihai S., Digest Journal of Nanomaterials and Biostructures, 2016, Vol. 11, No 1, pp. 271-276.

32.Boote B., Subramanian H., Ranjit K.T., Chemical communications, 2007, No 43, pp. 4543-4545.

33. Gaydhankar T.R., Taralkar U.S., Jha R.K., Joshi P.N. et al., Catalysis Communication, 2005, Vol. 6, Iss. 5, pp. 361-366.

Нефедова Татьяна Николаевна - старший научный сотрудник НИЦ «Фундаментальные исследования в области естественных и строительных наук имени Заслуженного деятеля нау-
34.Gallis K.W., Landry C.C, Chemistry of Materials, 1997, Vol. 9, Iss. 10, pp. 2035-2038.

35.Xia Y., Mokaya R., Titman J.J., J. Phys. Chem. B, 2004, Vol. 108, Iss. 31, pp. 1136111367.

36. Qin L., Zhao H., Wang H., Liu S., Advanced Materials Research, 2011, Vol. 306307, pp. 1420-1424.

37.Kim T.-W., Kleitz F., Paul B., Ryoo R., J. Am. Chem. Soc., 2005, Vol. 127, Iss. 20, pp. 7601-7610.

38.Gavalas G.R., Megiris C.E., Nam S.W., Chem. Eng. Sci., 1989, Vol. 44, Iss. 9, pp. 18291835.

39.Tsapatsis M., Kim S., Nam S.W., Gavalas G., Ind. Eng. Chem. Res., 1991, Vol. 30, Iss. 9, pp. 2152-2159.

40.Wang K., Lin Y., Morrisa M.A., Holmes J.D., J. Mater. Chem., 2006, Vol. 16, Iss. 41, pp. 4051-4057.

41.Duan Y., Shen Y., Water Science \& Technology, 2017, Vol. 76, Iss. 1, pp. 172-181.

42.Kim D.-J., Cho J.-K., Jang J.-H., Lee S.-C. et al., Applied Catalysis A: General, 2005, Vol. 286, Iss. 1, pp. 36-43.

43.Taba P., Mustafa R.D.P., Ramang L.M., Kasim A.H., Journal of Physics: Conf. Series, 2018, Vol. 979, Conference 1. ID. 012058.

44.Yuan Z.Y., Wang J.Z., Zhang Z.L., Chen T.H., Li H.X. // Microporous Mesoporous Mater. 2001. Vol. 43. Iss. 2. pp. 227-236.

45.Sun J.H., Shan Z., Maschmeyer T., Coppens M.O., Langmuir, 2003, Vol. 19, Iss. 20, pp. 8395-8402.

46. Grimes B.A., Lüdtke S., Unger K.K., Liapis A.I., Journal of Chromatography A, 2002, Vol. 979, Iss. 1-2, pp. 447-466.

47.Liapis A.I., Meyers J.J., Crosser O.K., Journal of Chromatography A, 1999, Vol. 865, Iss. 1-2, pp. 13-25.

48.Guan L.C., Nur H., Endud S., Journal of Physical Science, 2006, Vol. 17, Iss. 1, pp. 6575.

49.Jeon J.-K., Park H. J., Yim J.-H., Kim J. M. et al., Solid State Phenomena, 2007, Vol. 124126, pp. 1757-1760.

50.Schmuhl R., Chowdhury S.R., ten Elshof J.E., van den Berg A. et al., Journal of Sol-Gel Science and Technology, 2004, Vol. 31, Iss. 1-3, pp. 249-252.

Nefedova Tatiana N. - senior researcher SRC "Fundamental research in the fields of natural and building science named after the Honored Scientist of the Russian Federation, Professor Rossikhin Yuri 
ки РФ, профессора Россихина Юрия Алексеевича», Воронежский государственный технический университет, Воронеж

Ресснер Франк - профессор, зав. каф. технической химии 2, института химии, Ольденбургского университета имени Карла фон Осецкого, Ольденбург, Германия

Селеменев Владимир Федорович - профессор каф. аналитической химии, Воронежский государственный Университет, Воронеж
Alekseevich", Voronezh State Technical University, Voronezh, Russia; Postdoctoral Researcher at Department of Industrial Chemistry 2, Institute of Chemistry, Carl von Ossietzky University, Oldenburg, Germany, e-mail: TNephedova@gmail.com

Roessner Frank - professor, head of the Department of Industrial Chemistry 2, Institute of Chemistry, Carl von Ossietzky University, Oldenburg, Germany; e-mail: frank.roessner@unioldenburg.de

Selemenev Vladimir F.- professor of the department of Analytical Chemistry, faculty of Chemistry, Voronezh State University, Voronezh, Russia,e-mail: common@chem.vsu.ru 